PROCEEDINGS OF THE

AMERICAN MATHEMATICAL SOCIETY

Volume 130, Number 2, Pages 507-516

S 0002-9939(01)06294-3

Article electronically published on July 25, 2001

\title{
THE CONTINUOUS SPECTRUM OF THE DIRAC OPERATOR ON NONCOMPACT RIEMANNIAN SYMMETRIC SPACES OF RANK ONE
}

\author{
ROBERTO CAMPORESI AND EMMANUEL PEDON
}

(Communicated by Rebecca Herb)

\begin{abstract}
The continuous spectrum of the Dirac operator $D$ on the complex, quaternionic, and octonionic hyperbolic spaces is calculated using representation theory. It is proved that $\operatorname{spec}_{c}(D)=\mathbb{R}$, except for the complex hyperbolic spaces $H^{n}(\mathbb{C})$ with $n$ even, where $\operatorname{spec}_{c}(D)=\left(-\infty,-\frac{1}{2}\right] \cup\left[\frac{1}{2},+\infty\right)$.
\end{abstract}

\section{INTRODUCTION}

Noncompact Riemannian symmetric spaces $X=G / K$ are contractible manifolds and therefore possess a unique spin structure. One can thus define the Dirac operator $D$ acting on spinors on $X$ and study its $L^{2}$ spectrum $\operatorname{spec}(D)$. By general spectral theory, $\operatorname{spec}(D)$ is the reunion of the point (or discrete) $\operatorname{spectrum} \operatorname{spec}_{p}(D)$ and the continuous spectrum $\operatorname{spec}_{c}(D)$.

Recently Goette and Semmelmann calculated the point spectrum of the Dirac operator on all noncompact Riemannian symmetric spaces $X$. Their result is the following (see [8]): if $X$ is supposed irreducible, the $L^{2}$ point spectrum $\operatorname{spec}_{p}(D)$ of $D$ on $X$ is nonempty if and only if $X$ is isometric to $S U(p, q) / S(U(p) \times U(q))$ with $p+q$ odd, and in that $\operatorname{case} \operatorname{spec}_{p}(D)=\{0\}$.

It is also natural to wonder about the nature of the $L^{2}$ continuous spectrum $\operatorname{spec}_{c}(D)$ of $D$, especially since it appears, by the preceding result, that only a small class of noncompact Riemannian symmetric spaces carry a Dirac operator with nonempty point spectrum. In this paper, we shall prove the following result.

Theorem 1.1. If $X=G / K$ is a noncompact Riemannian symmetric space of rank one, then $\operatorname{spec}_{c}(D)=\mathbb{R}$, except if $X$ is a complex hyperbolic space $H^{n}(\mathbb{C})$ with $n$ even, in which case $\operatorname{spec}_{c}(D)=\left(-\infty,-\frac{1}{2}\right] \cup\left[\frac{1}{2},+\infty\right)$.

It is interesting to observe that the family of exceptional cases pointed out by this theorem (the spaces $X=H^{n}(\mathbb{C})$, with $n$ even) is precisely the one for which the Dirac operator has nonempty point spectrum. In other words, there is a spectral gap as soon as there is a point spectrum.

Received by the editors July $5,2000$.

2000 Mathematics Subject Classification. Primary 43A85, 58J50; Secondary 34L40, 53C27, $53 \mathrm{C} 35$.

Key words and phrases. Hyperbolic spaces, spinors, Dirac operator, spectral theory.

The second author was supported by the European Commission (TMR 1998-2001 Network Harmonic Analysis). 
Note that the result in the case of real hyperbolic spaces $H^{n}(\mathbb{R})$ was already known (see [6], [3], [7]). In the sequel, we shall therefore restrict our attention to the complex and quaternionic hyperbolic spaces $H^{n}(\mathbb{C}), H^{n}(\mathbb{H})$ (for $n \geq 2$ ), as well as to the octonionic plane $H^{2}(\mathbb{O})$.

\section{THE METHOD}

2.1. Some background material. The hyperbolic spaces $H^{n}(\mathbb{C}), H^{n}(\mathbb{H})$ (for $n \geq 2$ ) and $H^{2}(\mathbb{O})$ are manifolds of respective real dimension $d=2 n, d=4 n$ and $d=16$. We shall write them as $X=G / K$, where $G$ is respectively $S U(n, 1)$, $S p(n, 1)$ and $F_{4(-20)}$, and $K$ is respectively $S(U(n) \times U(1)), S p(n) \times S p(1)$ and $\operatorname{Spin}(9)$. Recall then that $G$ is a connected noncompact semisimple real Lie group with finite centre and that $K$ is a maximal compact subgroup of $G$.

Let $\mathfrak{g}, \mathfrak{k}$ be the Lie algebras of $G, K$, and let $\mathfrak{g}=\mathfrak{k} \oplus \mathfrak{p}$ be a fixed Cartan decomposition of $\mathfrak{g}$. Let the scalar product on $\mathfrak{p}$ be given by $\langle\cdot, \cdot\rangle=\left.c B\right|_{\mathfrak{p} \times \mathfrak{p}}$, where $B$ is the Killing form on $\mathfrak{g}$ and $c>0$ a normalization constant chosen so that this scalar product then induces the standard metric of sectional curvature $-4 \leq \kappa \leq-1$ on the hyperbolic spaces. When $\mathfrak{g}$ is linear, this convention gives $c B(X, Y)=\frac{1}{2} \operatorname{tr}(X Y)$ for any $X, Y \in \mathfrak{g}$.

Let $o=1_{G} K$ denote the origin in $G / K$, let Ad (resp. ad) denote the adjoint representation of $G$ (resp. of $\mathfrak{g}$ ), and consider the isotropy representation $\alpha$ of $K$ on $\mathfrak{p} \simeq T_{o}(G / K): \alpha:\left.k \mapsto \operatorname{Ad}(k)\right|_{\mathfrak{p}}$. By fixing an orthonormal basis $\left\{e_{i}\right\}_{i=1}^{d}$ of $\mathfrak{p}$, we can regard $\alpha$ as a homomorphism $\alpha: K \rightarrow S O(d)$, with differential $\alpha: \mathfrak{k} \rightarrow \mathfrak{s o}(d)$ given by $\alpha(Y)=\left.\operatorname{ad}(Y)\right|_{\mathfrak{p}}$.

In the quaternionic and octonionic cases, $K$ is simply connected and $\alpha$ lifts to a homomorphism $\tilde{\alpha}: K \rightarrow \operatorname{Spin}(d)$. In the complex case, $G$ and $K$ are not simply connected and $\alpha$ does not lift to $\operatorname{Spin}(d)$ if $n$ is even (see $\S 3$ ). In this case, we replace $G$ and $K$ by suitable double covers $\widetilde{G}$ and $\widetilde{K}$, so that $X=\widetilde{G} / \widetilde{K}$ and $\alpha$ lifts to a homomorphism $\tilde{\alpha}: \widetilde{K} \rightarrow \operatorname{Spin}(d)$. For simplicity, it will be understood in the remainder of this section that $G$ and $K$ have to be replaced by $\widetilde{G}$ and $\widetilde{K}$ in the even complex case.

Let $s$ be the complex spin representation of $\operatorname{Spin}(d)$ on $\mathbb{C}^{2^{d / 2}}$, with differential $s: \mathfrak{s o}(d) \simeq \mathfrak{s p i n}(d) \rightarrow \operatorname{End}\left(\mathbb{C}^{2^{d / 2}}\right)$ given by $s\left(E_{i j}-E_{j i}\right)=-\frac{1}{2} \gamma\left(e_{i}\right) \gamma\left(e_{j}\right)$, where $E_{i j}$ is the $d \times d$ matrix with entry 1 at place $(i, j)$ and zero entries elsewhere, and $\gamma: \mathbf{C l}(\mathfrak{p}) \rightarrow \operatorname{End}\left(\mathbb{C}^{2^{d / 2}}\right)$ denotes Clifford multiplication by elements of the complex Clifford algebra $\mathbf{C l}(\mathfrak{p})$ constructed from $\mathfrak{p}$.

The spin representation of $K$ is defined as the composite homomorphism $\tau=$ $s \circ \tilde{\alpha}$. It is a unitary representation of $K$ on $V_{\tau}=\mathbb{C}^{2^{d / 2}}$, with differential given explicitly by the formula

$$
\tau(Y)=s\left(\left.\operatorname{ad}(Y)\right|_{\mathfrak{p}}\right)=\frac{1}{4} \sum_{i, j}\left\langle\left[Y, e_{i}\right], e_{j}\right\rangle \gamma\left(e_{i}\right) \gamma\left(e_{j}\right)
$$

for $Y \in \mathfrak{k}$ (see [10], Lemma 2.1, or [4], §II.6.9).

The spinor bundle on $G / K$ can then be considered as the homogeneous vector bundle $E^{\tau}=G \times_{K} V_{\tau}$ associated with the principal bundle $G(G / K, K)$ and the representation $\tau$. The Levi-Civita connection on $X$ induces a connection on $E^{\tau}$, and the Dirac operator $D: \Gamma\left(E^{\tau}\right) \rightarrow \Gamma\left(E^{\tau}\right)$ is defined as usual on the space $\Gamma\left(E^{\tau}\right)$ of spinors on $E^{\tau}$ as the composition $\gamma \circ \nabla$ of the covariant derivative $\nabla$ with the fibrewise Clifford multiplication $\gamma$. 
2.2. The (continuous part of the) Plancherel formula for $L^{2}\left(E^{\tau}\right)$. Let $L^{2}\left(E^{\tau}\right)$ denote the Hilbert space of $L^{2}$ sections of $E^{\tau}$, i.e. of $L^{2}$ spinors on $X$. The first step towards the Plancherel formula for $L^{2}\left(E^{\tau}\right)$, which is the decomposition of $L^{2}\left(E^{\tau}\right)$ into $G$-irreducible constituents via an appropriate Fourier transform, consists of writing the decomposition of the spin representation $\tau$ into $K$-types (i.e. $K$-irreducible components).

Since $\mathfrak{g}$ and $\mathfrak{k}$ have equal complex rank, let $\mathfrak{h} \subset \mathfrak{k} \subset \mathfrak{g}$ be a common Cartan subalgebra. Denote by $\Delta_{\mathfrak{k}}=\Delta\left(\mathfrak{k}_{\mathbb{C}}, \mathfrak{h}_{\mathbb{C}}\right) \subset \Delta_{\mathfrak{g}}=\Delta\left(\mathfrak{g}_{\mathbb{C}}, \mathfrak{h}_{\mathbb{C}}\right)$ and by $W_{\mathfrak{k}}=W\left(\mathfrak{k}_{\mathbb{C}}, \mathfrak{h}_{\mathbb{C}}\right) \subset$ $W_{\mathfrak{g}}=W\left(\mathfrak{g}_{\mathbb{C}}, \mathfrak{h}_{\mathbb{C}}\right)$ the corresponding root systems and Weyl groups. Fix a system of positive roots $\Delta_{\mathfrak{g}}^{+}$in $\Delta_{\mathfrak{g}}$, and let $\Delta_{\mathfrak{k}}^{+}=\Delta_{\mathfrak{g}}^{+} \cap \Delta_{\mathfrak{k}}\left(\right.$ resp. $\left.\Delta_{\mathfrak{p}}^{+}=\Delta_{\mathfrak{g}}^{+} \backslash \Delta_{\mathfrak{k}}^{+}\right)$denote the subset of positive compact (resp. noncompact) roots. Define as usual the elements $\delta_{\mathfrak{g}}=\frac{1}{2} \sum_{\alpha \in \Delta_{\mathfrak{g}}^{+}} \alpha, \delta_{\mathfrak{k}}=\frac{1}{2} \sum_{\alpha \in \Delta_{\mathfrak{k}}^{+}} \alpha, \delta_{\mathfrak{p}}=\frac{1}{2} \sum_{\alpha \in \Delta_{\mathfrak{p}}^{+}} \alpha$. Let

$$
W^{1}=\left\{w \in W_{\mathfrak{g}}: w \Delta_{\mathfrak{g}}^{+} \supset \Delta_{\mathfrak{k}}^{+}\right\} .
$$

Let $\tau_{\lambda}$ denote the $K$-type with highest weight $\lambda$ relative to $\Delta_{\mathfrak{k}}^{+}$. It then follows from [10, Lemma 2.2, 4], Lemma II.6.9 or [12, Lemma 9.3.2, that

$$
\tau=\bigoplus_{w \in W^{1}} \tau_{w \delta_{\mathfrak{g}}-\delta_{\mathfrak{k}}}
$$

where each $K$-type with highest weight $w \delta_{\mathfrak{g}}-\delta_{\mathfrak{k}}$ occurs with multiplicity one. The map $W_{\mathfrak{k}} \times W^{1} \rightarrow W_{\mathfrak{g}}$ given by $(s, \sigma) \rightarrow s \cdot \sigma$ is a bijection, hence $W^{1} \simeq W_{\mathfrak{k}} \backslash W_{\mathfrak{g}}=$ $\left\{w_{j}, j \in J\right\}$, where $J$ is a finite set such that $|J|=\left|W_{\mathfrak{g}}\right| /\left|W_{\mathfrak{k}}\right|$ and the $w_{j}$ are distinguished representatives of $W_{\mathfrak{k}} \backslash W_{\mathfrak{g}}$ in $W_{\mathfrak{g}}$. Thus there are exactly $|J|$ systems of positive roots in $\Delta_{\mathfrak{g}}$ that contain $\Delta_{\mathfrak{k}}^{+}$, namely the $w_{j} \Delta_{\mathfrak{g}}^{+}$, with $j \in J$. Note that for all $w \in W^{1}, w \delta_{\mathfrak{g}}-\delta_{\mathfrak{k}}$ equals $\delta_{\mathfrak{p}}^{w \Delta_{\mathfrak{g}}^{+}}$, the half-sum of the noncompact roots of $w \Delta_{\mathfrak{g}}^{+}$. If $\tau_{j}$ has highest weight $\delta_{\mathfrak{p}}^{w_{j} \Delta_{\mathfrak{g}}^{+}}$, let us then rewrite (2.3) as

$$
\tau=\bigoplus_{j \in J} \tau_{j} .
$$

Now, let $\mathfrak{a} \subset \mathfrak{p}$ be a maximal abelian subspace, let $A=\exp (\mathfrak{a})$ be the corresponding real Lie group, and let $M$ be the centralizer of $A$ in $K$, with Lie algebra $\mathfrak{m}$. The second step consists of identifying the $M$-types that occur in the restriction to $M$ of each $K$-type $\tau_{j}$ of $\tau$. In other words, we look for a decomposition of the kind $\left.\tau_{j}\right|_{M}=\bigoplus_{\sigma \in \widehat{M}\left(\tau_{j}\right)} m\left(\sigma, \tau_{j}\right) \sigma$, where $\widehat{M}\left(\tau_{j}\right)$ denotes the set of $M$-types $\sigma$ that occur in $\left.\tau_{j}\right|_{M}$ with multiplicity $m\left(\sigma, \tau_{j}\right)>0$. To this end, we use the branching rules for $K \supset M$ given in [1] (and also in [2]). In the even complex case we actually need the branching rules for the double covers $\widetilde{K} \supset \widetilde{M}$, but these rules are formally the same as for $K \supset M$, since they are algebraic in nature and depend on the Lie algebras rather than the Lie groups.

By general representation theory for the real rank one semisimple Lie group $G$, the space $L^{2}\left(E^{\tau}\right)$ splits into the direct sum of its continuous part $L_{c}^{2}\left(E^{\tau}\right)$ and of its discrete part $L_{d}^{2}\left(E^{\tau}\right)$. For our purpose, we are only interested in the continuous part, that one can decompose as follows, by using Harish-Chandra's Plancherel Theorem for $L^{2}(G)$ (see e.g. [11], formula (3.3), for details):

$$
L_{c}^{2}\left(E^{\tau}\right) \simeq \bigoplus_{j \in J} \bigoplus_{\sigma \in \widehat{M}\left(\tau_{j}\right)} \int_{\mathbb{R}^{+}}^{\oplus} d \lambda p_{\sigma}(\lambda) H_{\sigma, \lambda} \otimes \operatorname{Hom}_{K}\left(H_{\sigma, \lambda}, V_{\tau_{j}}\right),
$$


where $H_{\sigma, \lambda}$ is the representation space of the unitary principal series $\pi_{\sigma, \lambda}$ of $G$, $d \lambda$ denotes Lebesgue measure on $\mathfrak{a}_{+}^{*} \simeq \mathbb{R}_{+}$and $p_{\sigma}(\lambda)$ is the Plancherel density associated with $\pi_{\sigma, \lambda}$. Note that the vector space $\operatorname{Hom}_{K}\left(H_{\sigma, \lambda}, V_{\tau_{j}}\right)$ of $K$-intertwining operators between $H_{\sigma, \lambda}$ and $V_{\tau_{j}}$ is isomorphic to $\mathbb{C}^{m\left(\sigma, \tau_{j}\right)}$ by Frobenius Reciprocity.

2.3. The Parthasarathy formula. For all spin manifolds, the celebrated Lichnerowicz formula relates the spinor Laplacian $D^{2}$ to the Bochner Laplacian $\nabla^{*} \nabla$ acting on smooth spinors. Namely,

$$
D^{2}=\nabla^{*} \nabla+\frac{S}{4} \mathrm{Id}
$$

where $S$ is the scalar curvature of $X$. With our previous convention on the metric on $X, S=-d\left(d+3 m_{2 \alpha}-1\right)$, where $m_{2 \alpha}$ is the multiplicity of the long positive restricted root $2 \alpha$ of the pair $(\mathfrak{g}, \mathfrak{a})$ and takes values 1,3 , and 7 for $H^{n}(\mathbb{C}), H^{n}(\mathbb{H})$, and $H^{2}(\mathbb{O})$, respectively. On the other hand, using Proposition 3.1 in [5] and our formula (2.1), we obtain the following Parthasarathy formula for the iterated Dirac operator $D^{2}$ on $X$ :

$$
D^{2}=-\Omega_{\mathfrak{g}}+\frac{S}{8} \mathrm{Id},
$$

where $\Omega_{\mathfrak{g}}$ is the Casimir operator associated with $\mathfrak{g}$, viewed here as a $G$-invariant differential operator acting on smooth sections of $E^{\tau}$.

2.4. The $L^{2}$ spectrum of $D$. By the Parthasarathy formula (2.6), it now suffices to read the effect of the Casimir operator $\Omega_{\mathfrak{g}}$ acting on the principal series representations $H_{\sigma, \lambda}$. By standard calculations (using for instance [9], Proposition 8.22 and Lemma 12.28) one easily checks that

$$
\pi_{\sigma, \lambda}\left(-\Omega_{\mathfrak{g}}\right)=\left[\lambda^{2}+\rho^{2}-c(\sigma)\right] \mathrm{Id}
$$

on $H_{\sigma, \lambda}$, where $\rho=\frac{1}{2}\left(d+m_{2 \alpha}-1\right)$ is half the sum of the positive restricted roots of $(\mathfrak{g}, \mathfrak{a})$, and $c(\sigma)>0$ is the Casimir value of $\sigma$ given by

$$
c(\sigma)=\left\|\mu_{\sigma}+\delta_{\mathfrak{m}}\right\|^{2}-\left\|\delta_{\mathfrak{m}}\right\|^{2}=\left\langle\mu_{\sigma}, \mu_{\sigma}+2 \delta_{\mathfrak{m}}\right\rangle
$$

if $\mu_{\sigma}$ and $\delta_{\mathfrak{m}}$ denote respectively the highest weight of $\sigma$ and half the sum of the positive roots in $\mathfrak{m}_{\mathbb{C}}$ relative to a fixed Cartan subalgebra.

Combining the above formulas we find

$$
\pi_{\sigma, \lambda}\left(D^{2}\right)=\left[\lambda^{2}+\rho^{2}-c(\sigma)+\frac{S}{8}\right] \mathrm{Id} .
$$

Now by (2.5) $\lambda \geq 0$ is arbitrary, and we just need to compute the non-negative quantity

$$
a=\inf \operatorname{spec}_{c}\left(D^{2}\right)=\rho^{2}+\frac{S}{8}-\sup \left\{c(\sigma): \sigma \in \widehat{M}\left(\tau_{j}\right), \tau_{j} \subset \tau\right\} .
$$

We have $\operatorname{spec}_{c}\left(D^{2}\right)=[a,+\infty)$ and $\operatorname{spec}_{c}(D)=(-\infty,-\sqrt{a}] \cup[\sqrt{a},+\infty)$, since $d$ is even and $\operatorname{spec}(D)$ is symmetric around zero.

Remark 2.1. In all cases, eigenspinors of the squared Dirac operator $D^{2}$ can be constructed as Poisson transforms of sections of a certain vector bundle over the boundary $G / P \simeq K / M$ of $X=G / K$. For details, see e.g. [11], $\S 4$, for the similar case of eigenforms of the Hodge-de Rham Laplacian on $X=H^{n}(\mathbb{C})$. 
3. The CASE $X=H^{n}(\mathbb{C})$

In this section, we take $X=H^{n}(\mathbb{C})$, with $n \geq 2$. Thus $G=S U(n, 1), \mathfrak{g}=$ $\mathfrak{s u}(n, 1), K=S(U(n) \times U(1))$ and $\mathfrak{k}=\mathfrak{s}(\mathfrak{u}(n) \oplus \mathfrak{u}(1))$, i.e.

$$
\mathfrak{k}=\left\{\left(\begin{array}{cc}
A & 0 \\
0 & b
\end{array}\right), \quad A \in \mathfrak{u}(n), \quad b \in \mathfrak{u}(1), \quad b+\operatorname{tr} A=0\right\} .
$$

We take

$$
\mathfrak{a}=\mathbb{R} \cdot\left(\begin{array}{ccc}
0 & 0 & 1 \\
0 & 0_{n-1} & 0 \\
1 & 0 & 0
\end{array}\right) \subset \mathfrak{p}
$$

so that

$$
\mathfrak{m}=\left\{\left(\begin{array}{ccc}
b & 0 & 0 \\
0 & A & 0 \\
0 & 0 & b
\end{array}\right), \quad A \in \mathfrak{u}(n-1), \quad b \in \mathfrak{u}(1), \quad 2 b+\operatorname{tr} A=0\right\} .
$$

As mentioned in the previous section, when $n$ is even we need to consider double covers $\widetilde{G}, \widetilde{K}$ and $\widetilde{M}$ of $G, K$ and $M$, respectively. However, for simplicity of exposition, we shall keep in the sequel the same notation for both the groups and their covers, except at the accurate place where these distinctions will be relevant (proof of next lemma).

Let $\mathfrak{h}$ (resp. $\mathfrak{t}$ ) denote the Cartan subalgebra of $\mathfrak{g}$ and $\mathfrak{k}$ (resp. of $\mathfrak{m}$ ) constituted with diagonal elements. For $1 \leq j \leq n+1$, let $\varepsilon_{j}$ be the linear functional on $\mathfrak{h}_{\mathbb{C}}$ defined by $\varepsilon_{j}\left(\operatorname{diag}\left(h_{1}, \ldots, h_{n+1}\right)\right)=h_{j}$. By abuse of notation, we shall keep the same denomination for the restriction of $\varepsilon_{j}$ to $\mathfrak{t}_{\mathbb{C}}$. The following choice of positive root systems is standard:

$$
\begin{aligned}
\Delta_{\mathfrak{g}}^{+} & =\left\{\varepsilon_{i}-\varepsilon_{j}, 1 \leq i<j \leq n+1\right\}, \\
\Delta_{\mathfrak{k}}^{+} & =\left\{\varepsilon_{i}-\varepsilon_{j}, 1 \leq i<j \leq n\right\}, \\
\Delta_{\mathfrak{m}}^{+} & =\left\{\varepsilon_{i}-\varepsilon_{j}, 2 \leq i<j \leq n\right\} .
\end{aligned}
$$

Let us decompose the spin representation $\tau$ of $K$. The subset $W^{1}$ of $W_{\mathfrak{g}}$ defined by (2.2) can be described as follows (see e.g. 2], $\S 11$ ). Denote by $s_{\beta}$ the reflection through the root $\beta$ and put

$$
w_{i}=\prod_{j=i+1}^{n} s_{\varepsilon_{j}-\varepsilon_{n+1}} \quad(0 \leq i \leq n-1), \quad w_{n}=\mathrm{id} .
$$

Then $W^{1}=\left\{w_{0}, \ldots, w_{n}\right\}$ and the half-sum of positive noncompact roots in $w_{i} \Delta_{\mathfrak{g}}^{+}$ equals

$$
\delta_{\mathfrak{p}}^{i}:=\delta_{\mathfrak{p}}^{w_{i} \Delta_{\mathfrak{g}}^{+}}=\frac{1}{2} \sum_{j=1}^{i} \varepsilon_{j}-\frac{1}{2} \sum_{j=i+1}^{n} \varepsilon_{j}+\left(\frac{n}{2}-i\right) \varepsilon_{n+1}
$$

for all $0 \leq i \leq n$ (it is understood that $\sum_{j=a}^{b}=0$ if $b<a$ ).

Let $\tau_{i}$ denote the $K$-type whose highest weight is $\delta_{\mathfrak{p}}^{i}$, so that (2.4) reads $\tau=$ $\bigoplus_{i=0}^{n} \tau_{i}$.

Lemma 3.1. We have the decompositions

$$
\left.\tau_{0}\right|_{M}=\sigma_{0},\left.\quad \tau_{n}\right|_{M}=\sigma_{n-1},\left.\quad \tau_{i}\right|_{M}=\sigma_{i-1} \oplus \sigma_{i} \quad(1 \leq i \leq n-1),
$$


where, for $0 \leq i \leq n-1, \sigma_{i}$ is the $M$-type with highest weight

$$
\mu_{i}=\frac{n-1-2 i}{4}\left(\varepsilon_{1}+\varepsilon_{n+1}\right)+\frac{1}{2} \sum_{j=2}^{i+1} \varepsilon_{j}-\frac{1}{2} \sum_{j=i+2}^{n} \varepsilon_{j}
$$

and occurs with multiplicity 1 .

Proof. In the following discussion, it becomes necessary to distinguish $K$ (resp. $M$ ) from $\widetilde{K}$ (resp. $\widetilde{M}$ ). Let us recall first the branching rule given in [1], Theorem 4.4 (see also [2], Theorem 10.5). Let $\lambda=\sum_{j=1}^{n+1} a_{j} \varepsilon_{j}$, with $a_{1} \geq \cdots \geq a_{n}$ and $a_{j} \in \mathbb{Z}$ for all $j$, be the highest weight of $\tau_{\lambda} \in \widehat{K}$. Let $\mu=b_{0}\left(\varepsilon_{1}+\varepsilon_{n+1}\right)+\sum_{j=2}^{n} b_{j} \varepsilon_{j}$, with $b_{2} \geq \cdots \geq b_{n}, 2 b_{0} \in \mathbb{Z}$ and $b_{j} \in \mathbb{Z}$ for all $j \geq 1$, be the highest weight of $\sigma_{\mu} \in \widehat{M}$. Then $\left.\tau_{\lambda}\right|_{M} \supset \sigma_{\mu}$ if and only if

$$
\begin{cases}a_{1} \geq b_{2} \geq \cdots \geq b_{n} \geq a_{n} & \text { and } \\ b_{0}=\left(b_{1}+a_{n+1}\right) / 2 & \text { where } b_{1}=a_{1}+\sum_{j=2}^{n}\left(a_{j}-b_{j}\right),\end{cases}
$$

and in that case, the multiplicity always equals 1 .

Our representation $\tau_{i}$ has highest weight $\delta_{\mathfrak{p}}^{i}$, which at first glance does not belong to $\widehat{K}$, since its coefficients $a_{j}$ are half-integers instead of being integers. But a simple verification shows that the weight $\delta_{\mathfrak{p}}^{i}$ is actually analytically integral if $n$ is odd, so that the corresponding representation of $\mathfrak{k}$ indeed lifts to a representation of $K$ in that case. The explanation is that we can (and must) add the quantity $\frac{1}{2} \sum \varepsilon_{j}=0$ to $\delta_{\mathfrak{p}}^{i}$ in order to match the parametrization of $\widehat{K}$ given above. Since the same argument works for the weight $\mu_{i}$ of $M$ defined in the statement, the decompositions follow by the branching rule.

On the contrary, in the even case, $\delta_{\mathfrak{p}}^{i}\left(\right.$ resp. $\mu_{i}$ ) is never a highest weight of $K$ (resp. of $M$ ). However $2 \delta_{\mathfrak{p}}^{i}$ (resp. $2 \mu_{i}$ ) is such a weight of $K$ (resp. of $M$ ), and this explains why it is necessary and sufficient in that case to take double coverings of the groups involved. Besides, since the branching rule depends only on the Lie algebras, the decompositions are the same.

It is now an easy matter to calculate the Casimir values $c\left(\sigma_{i}\right)$ of the $M$-types $\sigma_{i}(0 \leq i \leq n-1)$ by formula (2.7). In order to match our normalization of the curvature, we must take

$$
\left\langle\varepsilon_{i}, \varepsilon_{j}\right\rangle= \begin{cases}\frac{2 n}{n+1} & \text { if } i=j \\ \frac{-2}{n+1} & \text { if } i \neq j\end{cases}
$$

We then obtain

$$
c\left(\sigma_{i}\right)=\left\langle\mu_{i}+2 \delta_{\mathfrak{m}}, \mu_{i}\right\rangle=\frac{n^{2}-1}{4}+i(n-i-1), \quad 0 \leq i \leq n-1 .
$$

On the other hand, we have in our situation $\rho=n$ and $S=-4 n(n+1)$, so that

$$
\rho^{2}+\frac{S}{8}-c\left(\sigma_{i}\right)=\left(\frac{n-1}{2}-i\right)^{2}, \quad 0 \leq i \leq n-1 .
$$

The lowest values of these quantities are attained for $i=\frac{n}{2}-1$ and $i=\frac{n}{2}$ if $n$ is even, and for $i=\frac{n-1}{2}$ if $n$ is odd, and we get

$$
a=\inf \operatorname{spec}_{c}\left(D^{2}\right)= \begin{cases}\frac{1}{4} & \text { if } n \text { is even } \\ 0 & \text { if } n \text { is odd }\end{cases}
$$


Thus we have proved the following:

Proposition 3.2. If $X=H^{n}(\mathbb{C})$, then

$$
\operatorname{spec}_{c}(D)= \begin{cases}\left(-\infty,-\frac{1}{2}\right] \cup\left[\frac{1}{2},+\infty\right) & \text { if } n \text { is even } \\ \mathbb{R} & \text { if } n \text { is odd }\end{cases}
$$

\section{THE CASE $X=H^{n}(\mathbb{H})$}

We now consider $X=H^{n}(\mathbb{H})$, with $n \geq 2$. Then $G=S p(n, 1), \mathfrak{g}=\mathfrak{s p}(n, 1)$ and $K=S p(n) \times S p(1)$, whose Lie algebra $\mathfrak{k}=\mathfrak{s p}(n) \oplus \mathfrak{s p}(1)$ can be realized in the following manner:

$$
\mathfrak{k}=\left\{\left(\begin{array}{cccc}
A & 0 & B & 0 \\
0 & u & 0 & v \\
-\bar{B} & 0 & \bar{A} & 0 \\
0 & -\bar{v} & 0 & \bar{u}
\end{array}\right), \quad \begin{array}{l}
A \in \mathfrak{u}(n), B \in M(n, \mathbb{C}), \\
B \text { symmetric, } u \in \mathfrak{u}(1), v \in \mathbb{C}
\end{array}\right\}
$$

Let

$$
\mathfrak{p}=\left\{\left(\begin{array}{cccc}
0 & c & 0 & d \\
\bar{c}^{t} & 0 & d^{t} & 0 \\
0 & \bar{d} & 0 & -\bar{c} \\
\bar{d}^{t} & 0 & -c^{t} & 0
\end{array}\right), c, d \in \mathbb{C}^{n}\right\}
$$

We take $\mathfrak{a}=\mathbb{R} H$, where $H \in \mathfrak{p}$ has $c^{t}=(1,0, \ldots, 0)$ and $d=0$ in the parametrization above. With this choice,

$$
\begin{aligned}
\mathfrak{m} & \left.=\left\{\begin{array}{cccccc}
u & 0 & 0 & -v & 0 & 0 \\
0 & A & 0 & 0 & B & 0 \\
0 & 0 & u & 0 & 0 & v \\
\bar{v} & 0 & 0 & \bar{u} & 0 & 0 \\
0 & -\bar{B} & 0 & 0 & \bar{A} & 0 \\
0 & 0 & -\bar{v} & 0 & 0 & \bar{u}
\end{array}\right), \begin{array}{l}
A \in \mathfrak{u}(n-1), B \in M(n-1, \mathbb{C}), \\
B \text { symmetric, } u \in \mathfrak{u}(1), v \in \mathbb{C}
\end{array}\right\} \\
& \simeq \mathfrak{s p}(n-1) \oplus \mathfrak{s p}(1) .
\end{aligned}
$$

Let $\mathfrak{h}$ (resp. $\mathfrak{t}$ ) be the Cartan subalgebra of $\mathfrak{g}$ and $\mathfrak{k}$ (resp. of $\mathfrak{m}$ ) constituted with diagonal elements. For $1 \leq j \leq n+1$, let $E_{j} \in \mathfrak{h}_{\mathbb{C}}$ denote the matrix defined by $\left(E_{j}\right)_{i i}=1$ if $i=j,\left(E_{j}\right)_{i i}=-1$ if $i=n+1+j$ and $\left(E_{j}\right)_{i i}=0$ otherwise. Then $\left\{E_{j}\right\}$ is a basis of $\mathfrak{h}_{\mathbb{C}}$. Denote by $\left\{\varepsilon_{j}\right\}$ the corresponding dual basis of $\mathfrak{h}_{\mathbb{C}}^{*}$. As before, we keep the same notation for the restriction of $\varepsilon_{j}$ to $\mathfrak{t}_{\mathbb{C}}$. We take:

$$
\begin{aligned}
\Delta_{\mathfrak{g}}^{+} & =\left\{\varepsilon_{i} \pm \varepsilon_{j}, 1 \leq i<j \leq n+1\right\} \cup\left\{2 \varepsilon_{k}, 1 \leq k \leq n+1\right\}, \\
\Delta_{\mathfrak{k}}^{+} & =\left\{\varepsilon_{i} \pm \varepsilon_{j}, 1 \leq i<j \leq n\right\} \cup\left\{2 \varepsilon_{k}, 1 \leq k \leq n+1\right\}, \\
\Delta_{\mathfrak{m}}^{+} & =\left\{\varepsilon_{1}+\varepsilon_{n+1} ; \varepsilon_{i} \pm \varepsilon_{j}, 2 \leq i<j \leq n\right\} \cup\left\{2 \varepsilon_{k}, 2 \leq k \leq n\right\} .
\end{aligned}
$$

As in the previous case, we use [2], §11, to see that the spin representation $\tau$ of $K$ decomposes as $\tau=\bigoplus_{i=0}^{n} \tau_{i}$, where $\tau_{i}$ is the $K$-type with highest weight $\delta_{\mathfrak{p}}^{i}=\sum_{j=1}^{i} \varepsilon_{j}+(n-i) \varepsilon_{n+1}$. We now give the $M$-decompositions of these $K$-types.

Lemma 4.1. Let $\sigma_{i}$ and $\sigma_{i}^{\prime}$ be the $M$-types having respectively as highest weights

$$
\mu_{i}=\frac{n-i}{2}\left(\varepsilon_{1}+\varepsilon_{n+1}\right)+\sum_{j=2}^{i+1} \varepsilon_{j} \quad \text { and } \quad \mu_{i}^{\prime}=\frac{n-i-1}{2}\left(\varepsilon_{1}+\varepsilon_{n+1}\right)+\sum_{j=2}^{i} \varepsilon_{j} .
$$

Then we have the following (multiplicity free) decompositions: 
(i) if $i=0,\left.\tau_{0}\right|_{M}=\sigma_{0}$;

(ii) if $i=1,\left.\tau_{1}\right|_{M}=\sigma_{1} \oplus \sigma_{1}^{\prime} \oplus \sigma_{0}$;

(iii) if $2 \leq i \leq n-1,\left.\tau_{i}\right|_{M}=\sigma_{i} \oplus \sigma_{i}^{\prime} \oplus \sigma_{i-1} \oplus \sigma_{i-1}^{\prime}$;

(iv) if $i=n,\left.\tau_{n}\right|_{M}=\sigma_{n} \oplus \sigma_{n-1}^{\prime}$.

Proof. For convenience of the reader, let us recall the branching rule given in [1, Theorem 5.5 (see also [2], Theorem 10.7). Let $\lambda=\sum_{j=1}^{n+1} a_{j} \varepsilon_{j}$, with $a_{1} \geq \cdots \geq a_{n}$ and $a_{j} \in \mathbb{N}$ for all $j$, be the highest weight of $\tau_{\lambda} \in \widehat{K}$. Let $\mu=b_{0}\left(\varepsilon_{1}+\varepsilon_{n+1}\right)+$ $\sum_{j=2}^{n} b_{j} \varepsilon_{j}$, with $2 b_{0} \in \mathbb{N}, b_{2} \geq \cdots \geq b_{n}$ and $b_{j} \in \mathbb{N}$ for all $j \geq 2$, be the highest weight of $\sigma_{\mu} \in \widehat{M}$. Define

$$
\begin{aligned}
& A_{1}=a_{1}-\max \left(a_{2}, b_{2}\right), \quad A_{2}=\min \left(a_{2}, b_{2}\right)-\max \left(a_{3}, b_{3}\right), \quad \ldots \\
& A_{n-1}=\min \left(a_{n-1}, b_{n-1}\right)-\max \left(a_{n}, b_{n}\right), \quad A_{n}=\min \left(a_{n}, b_{n}\right) .
\end{aligned}
$$

Then $\left.\tau_{\lambda}\right|_{M} \supset \sigma_{\mu}$ if and only if

(1) $a_{j} \geq b_{j+1}$ for $1 \leq j \leq n-1$,

(2) $b_{j} \geq a_{j+1}$ for $2 \leq j \leq n-1$ and

(3) $b_{0}=\frac{a_{n+1}+b_{1}-2 l}{2}$ for some $l \in\left\{0, \ldots, \min \left(a_{n+1}, b_{1}\right)\right\}$,

where $b_{1}$ satisfies $b_{1} \in \mathbb{N}$ and $\sum_{j=1}^{n}\left(a_{j}+b_{j}\right) \in 2 \mathbb{N}$. If these conditions hold, then the multiplicity of $\sigma_{\mu}$ in $\left.\tau_{\lambda}\right|_{M}$ equals $m(\mu, \lambda):=\sum_{b_{1} \text { satisfying (3) }} \widetilde{m}(\mu, \lambda)$, where

$$
\widetilde{m}(\mu, \lambda)=\sum_{L \subset\{1, \ldots, n\}}(-1)^{|L|}\left(\begin{array}{c}
n-2-|L|+\frac{1}{2}\left(-b_{1}+\sum_{j=1}^{n} A_{j}\right)-\sum_{j \in L} A_{j} \\
n-2
\end{array}\right) .
$$

$\left(|L|\right.$ is the cardinality of $L$ and by convention $\left(\begin{array}{l}x \\ y\end{array}\right)=0$ if $x-y \notin \mathbb{N}$.)

For simplicity, we shall give the proof of the decompositions claimed above in the case $3 \leq i \leq n-1$ (the proofs are similar in the other cases). For our $K$ type $\tau_{i}$, we have $a_{1}=\cdots=a_{i}=1, a_{i+1}=\cdots=a_{n}=0$ and $a_{n+1}=n-i$. Assume that $\sigma_{\mu}$ occurs in $\left.\tau_{i}\right|_{M}$. Then the conditions (1) and (2) imply the relations $b_{2}=\cdots=b_{i-1}=1, b_{i+2}=\cdots b_{n}=0$ and $1 \geq b_{i} \geq b_{i+1} \geq 0$. Therefore three cases must be distinguished.

First case: $b_{i}=b_{i+1}=1$. Then $b_{1}=2 k$ for some $k \in \mathbb{N}$ and $A_{1}=\cdots=A_{n}=0$. The condition $\left(\begin{array}{c}n-2-|L|-k \\ n-2\end{array}\right) \neq 0$ forces $k=|L|=0$, hence $b_{1}=0$ and, by (3), one has $b_{0}=\frac{n-i}{2}$, which gives $\sigma_{\mu}=\sigma_{i}$.

Second case: $b_{i}=1$ and $b_{i+1}=0$. Then $b_{1}=2 k+1$ for some $k \in \mathbb{N}, A_{i}=1$ and $A_{j}=0$ for all $j \neq i$. Then

$$
\widetilde{m}(\mu, \lambda)=\sum_{L \ni i}(-1)^{|L|}\left(\begin{array}{c}
n-2-|L|-k-1 \\
n-2
\end{array}\right)+\sum_{L \not \supset i}(-1)^{|L|}\left(\begin{array}{c}
n-2-|L|-k \\
n-2
\end{array}\right) .
$$

The first term in the right-hand side above is zero, therefore $\widetilde{m}(\mu, \lambda) \neq 0$ forces $k=|L|=0$ and $b_{1}=1$. Thus $b_{0}=\frac{n-i \pm 1}{2}$ and $\sigma_{\mu}$ can either be $\sigma_{i}^{\prime}$ or $\sigma_{i-1}$.

Third case: $b_{i}=b_{i+1}=0$. In this case, $b_{1}=2 k$ for some $k \in \mathbb{N}$ and $A_{1}=$ $\cdots=A_{n}=0$. As in the first case, one sees that $b_{1}=0$ and $b_{0}=\frac{n-i}{2}$, which yields $\sigma_{\mu}=\sigma_{i-1}^{\prime}$.

Let us now identify the highest Casimir value $\sup c(\sigma)$ of the $M$-types $\sigma$ occuring in $\left.\tau_{i}\right|_{M}$ for all $i$. A first examination shows that it suffices, for a fixed $i$, to compare $c\left(\sigma_{i}\right)$ with $c\left(\sigma_{i-1}\right)$. An easy calculation using the formula $\left\langle\varepsilon_{i}, \varepsilon_{j}\right\rangle=2 \delta_{i j}$ (adapted to our curvature normalization) then gives

$$
c\left(\sigma_{i}\right)=(n-i)(n+i+2)+2 i n, \quad c\left(\sigma_{i-1}\right)=c\left(\sigma_{i}\right)+2(i-n)+1,
$$


hence $c\left(\sigma_{i}\right) \geq c\left(\sigma_{i-1}\right)$ unless $i=n$. Therefore,

$$
\sup \left\{c(\sigma):\left.\sigma \subset \tau_{i}\right|_{M}\right\}= \begin{cases}c\left(\sigma_{i}\right)=(n-i)(n+i+2)+2 i n & \text { if } 0 \leq i \leq n-1 \\ c\left(\sigma_{n-1}\right)=2 n^{2}+1 & \text { if } i=n\end{cases}
$$

It is clear that the highest value of these numbers is $c\left(\sigma_{n-1}\right)$, which is attained both for $i=n-1$ and for $i=n$. Since in our setting $\rho=2 n+1$ and $S=-16 n(n+2)$, we get $a=\rho^{2}+\frac{S}{8}-c\left(\sigma_{n-1}\right)=0$.

Thus we have proved the following result.

Proposition 4.2. If $X=H^{n}(\mathbb{H})$, then $\operatorname{spec}_{c}(D)=\mathbb{R}$.

\section{THE CASE $H^{2}(\mathbb{O})$}

The remaining case is the one of the octonionic plane $X=H^{2}(\mathbb{O})$. We have here $G=F_{4(-20)}$ and $K=\operatorname{Spin}(9)$.

In this setting, the algebraic structure is more complicated than in the two previous cases, and we have no place here to introduce all of the notations necessary to state and use the branching rule from $K$ to $M \simeq \operatorname{Spin}(7)$. For this reason, we shall simply list below the main results, without proofs. A patient reader will easily retrieve the arguments, with the help of [1], $\S 6$ and/or [2], $\S \S 10.8 \& 11.20$ (see also the proof of Lemma 15.9).

Thus let us admit that the root systems can be written as

$$
\begin{aligned}
\Delta_{\mathfrak{g}} & =\left\{ \pm \varepsilon_{i}, 1 \leq i \leq 4 ; \pm \varepsilon_{i} \pm \varepsilon_{j}, 1 \leq i<j \leq 4, \frac{1}{2}\left( \pm \varepsilon_{1} \pm \varepsilon_{2} \pm \varepsilon_{3} \pm \varepsilon_{4}\right)\right\}, \\
\Delta_{\mathfrak{k}} & =\left\{ \pm \varepsilon_{i}, 1 \leq i \leq 4 ; \pm \varepsilon_{i} \pm \varepsilon_{j}, 1 \leq i<j \leq 4\right\} \\
\Delta_{\mathfrak{m}} & =\left\{ \pm \varepsilon_{i}, 2 \leq i \leq 4 ; \pm \varepsilon_{i} \pm \varepsilon_{j}, 2 \leq i<j \leq 4\right\} .
\end{aligned}
$$

The decomposition (2.4) of the spin representation of $K$ is $\tau=\tau_{1} \oplus \tau_{2} \oplus \tau_{3}$, where the occuring $K$-types $\tau_{i}(i=1,2,3)$ have respective highest weights

$$
\lambda_{1}=\varepsilon_{1}+\varepsilon_{2}+\varepsilon_{3}, \quad \lambda_{2}=\frac{3}{2} \varepsilon_{1}+\frac{1}{2} \varepsilon_{2}+\frac{1}{2} \varepsilon_{3}+\frac{1}{2} \varepsilon_{4}, \quad \lambda_{3}=2 \varepsilon_{1} .
$$

Lemma 5.1. (i) The $M$-types of $\left.\tau_{1}\right|_{M}$ are $\sigma_{1}^{j}(j=1, \ldots, 4)$, having as respective highest weights

$$
\mu_{1}^{1}=\frac{3}{2} \varepsilon_{2}+\frac{1}{2} \varepsilon_{3}+\frac{1}{2} \varepsilon_{4}, \quad \mu_{1}^{2}=\frac{1}{2} \varepsilon_{2}+\frac{1}{2} \varepsilon_{3}+\frac{1}{2} \varepsilon_{4}, \quad \mu_{1}^{3}=\varepsilon_{2}+\varepsilon_{3}, \quad \mu_{1}^{4}=\varepsilon_{2} .
$$

All occur with multiplicity 1.

(ii) The $M$-types of $\left.\tau_{2}\right|_{M}$ are $\sigma_{2}^{j}(j=1, \ldots, 6)$, having as respective highest weights

$$
\begin{aligned}
& \mu_{2}^{1}=\varepsilon_{2}+\varepsilon_{3}+\varepsilon_{4}, \quad \mu_{2}^{2}=\varepsilon_{2}+\varepsilon_{3}, \quad \mu_{2}^{3}=\frac{3}{2} \varepsilon_{2}+\frac{1}{2} \varepsilon_{3}+\frac{1}{2} \varepsilon_{4}, \\
& \mu_{2}^{4}=\frac{1}{2} \varepsilon_{2}+\frac{1}{2} \varepsilon_{3}+\frac{1}{2} \varepsilon_{4}, \quad \mu_{2}^{5}=\varepsilon_{2}, \quad \mu_{2}^{6}=0 .
\end{aligned}
$$

All occur with multiplicity 1 , except $\mu_{2}^{4}$ which appears with multiplicity 2.

(iii) The $M$-types of $\left.\tau_{3}\right|_{M}$ are $\sigma_{3}^{j}(j=1,2,3)$, having as respective highest weights:

$$
\mu_{3}^{1}=\varepsilon_{2}+\varepsilon_{3}+\varepsilon_{4}, \quad \mu_{3}^{2}=\frac{1}{2} \varepsilon_{2}+\frac{1}{2} \varepsilon_{3}+\frac{1}{2} \varepsilon_{4}, \quad \mu_{3}^{3}=0 .
$$

All occur with multiplicity 1. 
A quick comparison between the Casimir values of the $M$-types (using $\left\langle\varepsilon_{i}, \varepsilon_{j}\right\rangle=$ $4 \delta_{i j}$ and $\left.2 \delta_{\mathfrak{m}}=5 \varepsilon_{2}+3 \varepsilon_{3}+\varepsilon_{4}\right)$ shows that

$$
\sup \left\{c(\sigma):\left.\sigma \subset \tau_{i}\right|_{M}\right\}= \begin{cases}c\left(\sigma_{1}^{1}\right)=49 & \text { if } i=1, \\ c\left(\sigma_{2}^{3}\right)=49 & \text { if } i=2, \\ c\left(\sigma_{3}^{1}\right)=48 & \text { if } i=3 .\end{cases}
$$

Now we use the values $\rho=11$ and $S=-576$ to see that $a=\rho^{2}+\frac{S}{8}-49=0$. Hence we have proved the following result and, ultimately, the theorem claimed in the introduction of our article.

Proposition 5.2. If $X=H^{2}(\mathbb{O})$, then $\operatorname{spec}_{c}(D)=\mathbb{R}$.

\section{REFERENCES}

1. M. W. Baldoni-Silva, Branching theorems for semisimple Lie groups of real rank one, Rend. Sem. Mat. Univ. Padova 61 (1979), 229-250. MR 83a:22012

2. $ـ$, The embeddings of the discrete series in the principal series for semisimple Lie groups of real rank one, Trans. Amer. Math. Soc. 261 (1980), 303-369. MR 82b:22022

3. C. Bär, The Dirac operator on hyperbolic manifolds of finite volume, Preprint, Universität Freiburg, 1998.

4. A. Borel and N. R. Wallach, Continuous Cohomology, Discrete Subgroups, and Representations of Reductive Groups, second ed., Math. Surveys Monogr. 67, Amer. Math. Soc., Providence, RI, 2000. MR 2000j:22015

5. T. P. Branson, G. Ólafsson, and H. Schlichtkrull, A bundle-valued Radon transform, with applications to invariant wave equations, Quart. J. Math. Oxford (2) 45 (1994), 429-461. MR 95k:22020

6. U. Bunke, The spectrum of the Dirac operator on the hyperbolic space, Math. Nachr. 153 (1991), 179-190. MR 92h:58196

7. R. Camporesi and E. Pedon, Harmonic analysis for spinors on real hyperbolic spaces, Colloq. Math. 87 (2001), 245-286. CMP 2001:08

8. S. Goette and U. Semmelmann, The point spectrum of the Dirac operator on noncompact symmetric spaces, Preprint, 1999. To appear in Proc. Amer. Math. Soc.

9. A. W. Knapp, Representation Theory of Semisimple Groups. An Overview Based on Examples, Princeton Math. Ser. 36, Princeton Univ. Press, Princeton, NJ, 1986. MR 87j:22022

10. R. Parthasarathy, Dirac operator and the discrete series, Ann. of Math. 96 (1972), 1-30. MR 47:6945

11. E. Pedon, Harmonic analysis for differential forms on complex hyperbolic spaces, J. Geom. Phys. 32 (1999), 102-130. MR 2000j:22013

12. N. R. Wallach, Real Reductive Groups I, Academic Press, New York, 1988. MR 89i:22029

Dipartimento di Matematica, Politecnico di Torino, Corso Duca degli Abruzzi 24, 10129 TORINO, ITALY

E-mail address: camporesi@polito.it

Laboratoire de Mathématiques, Université de Reims, UPRESA 6056, Moulin de la Housse, B.P. 1039, 51687 Reims Cedex 2, France

E-mail address: emmanuel.pedon@univ-reims.fr 